\title{
Early Onset Peritoneal Dialysis-Related Peritonitis
}

\section{Samantha $\mathrm{Ng}^{1}$, David W Johnson ${ }^{2,3,4 *}$ and Yeoungjee Cho ${ }^{2,3,4}$}

${ }^{1}$ Department of Nephrology, Logan Hospital, Australia

${ }^{2}$ Department of Nephrology, Princess Alexandra Hospital, Brisbane, Australia

${ }^{3}$ Australasian Kidney Trials Network, University of Queensland, Brisbane, Australia

${ }^{4}$ Translational Research Institute, Brisbane, Australia

*Corresponding author: Professor David W Johnson, Department of Nephrology, Princess Alexandra Hospital, 199 Ipswich Road, Woolloongabba, Brisbane, QLD-4102, Australia, Tel: +61 73176 5080; Fax: +61 73176 5480; E-mail: david.johnson2@health.qld.gov.au

Received date: June 22, 2017; Accepted date: July 14, 2017; Published date: July 20, 2017

Citation: Ng S, Johnson DW, Cho Y (2017) Early Onset Peritoneal Dialysis-Related Peritonitis. J Clin Exp Nephrol Vol.2 No.3: 44.

Copyright: (C2017 Ng S, et al. This is an open-access article distributed under the terms of the Creative Commons Attribution License, which permits unrestricted use, distribution, and reproduction in any medium, provided the original author and source are credited.

\section{Abstract}

Peritonitis is a serious and common complication of peritoneal dialysis (PD), representing the commonest cause of technique failure and a not infrequent cause of patient death. Early onset peritonitis, variously defined in the literature as peritonitis occurring within the first 3-20 months of PD, is increasingly being recognised as a discrete and important clinical problem with appreciably heightened risks of technique failure and death. The aim of this review is to provide a comprehensive up to date summary of current evidence surrounding early onset peritonitis and to discuss how to better manage the condition, including suggestions for future research directions.

Keywords: Early onset peritonitis; Outcomes; Peritonitis; Peritoneal dialysis; Risk factors

\section{Introduction}

Peritonitis is a common and serious complication of peritoneal dialysis (PD) with reported rates ranging from 0.06 to 1.66 episodes per patient year across different countries [1]. It directly contributes to approximately $20 \%$ of technique failures and up to $6 \%$ of deaths in PD patients [2]. Moreover, severe or persistent peritonitis can lead to peritoneal membrane injury, possibly promoting the development of encapsulating peritoneal sclerosis $[3,4]$. PD peritonitis has thus been a strong focus of clinical research and there have been many initiatives implemented to reduce the burden of peritonitis, including better identification of risk factors [4]. However, it remains uncertain whether these risk factors for peritonitis are relevant for all episodes regardless of their onset or whether there may be differences depending on the timing of peritonitis occurrence.

Early onset peritonitis (EOP) is a term which has been described with variable definitions. Referred to as time of onset of first episode of peritonitis after PD commencement, various definitions have been proposed from 90 days to 20.28 months after PD onset [4-11]. There has been a growing interest in this topic as patients with early onset peritonitis appear to be placed at a heightened risk of complications, including technique failure and all-cause mortality, when compared to those who develop peritonitis later in their PD treatment [4-6]. This review will summarise the state of evidence to date on early onset peritonitis, its definition, importance, incidence, risk factors and preventative strategies.

\section{Definition of EOP}

In defining EOP, it is important to first examine the incidence of peritonitis in general. Reported rates of peritonitis vary widely worldwide, from 0.06 episodes per patient year in Taiwan to 1.66 episodes per patient year in Israel [7]. The hypothesis that there are differences in outcomes across patient groups according to time of onset of peritonitis has been suggested as early as 2003 [8]. Overall, it has been postulated that EOP occurs in up to $30 \%$ of PD patients $[5,7]$. However, definitions of "early" have varied significantly across studies, with time points proposed at as early as 3 months $[6,9,10]$ and as late as almost 2 years after initiation of PD [11]. The majority of these studies have been single-centre, retrospective observational studies of relatively small patient numbers, and the definition of 'early' was chosen by the investigators with no justification for how this was determined $[5,6,8,10]$. Hsieh and colleagues from Taiwan proposed a definition based on median time to first episode of peritonitis in their cohort, which was 20.28 months in their study [11]. This may be almost as long as median PD technique survival in some countries, such as Australia and New Zealand [12]. Most commonly, the definition of 'early' in EOP has been 3 months (or 90 days) [5,10,11], followed equally by $6[5,7]$ and 12 months $[7,13]$.

\section{Incidence of EOP}

Determining the incidence of EOP has been hampered by variable definitions, particularly with respect to $\mathrm{PD}$ commencement and whether this encompasses PD catheter 
insertion or commencement of dialysis. The risk of peritonitis is continuous and starts from when a PD catheter is inserted. In the literature to date, EOP has been generally defined as peritonitis occurring within a specified time period (i.e., 3 months, 6 months) from PD commencement, but definitions have variably included episodes after PD catheter insertion (i.e. inclusive of the period prior to PD commencement). Understanding these differences can be important when trying to determine the incidence of EOP and compare results across studies.

One of the earliest studies conducted was by $\mathrm{Wu}$ et al. in which EOP, defined as any peritonitis occurring within 3 months of PD commencement, was evaluated in a retrospective observational cohort study of 1690 incident patients followed up for a median period of 32.6 months at a single Chinese centre [9]. After excluding 87 patients who ceased PD within 3 months (for reasons other than peritonitis), EOP was observed to occur in 118 (7\%) patients. An important limitation of this study was exclusion of patients who had been on PD for less than three months, which may have introduced selection bias. The single centre design also limited generalizability of the study's findings.

A subsequent retrospective observational study by Vargas et al. examined EOP within 90 days of treatment commencement in 3525 incident PD patients across 49 PD centres belonging to a single dialysis provider in Colombia [6]. The incidence of EOP was reported as 0.23 episodes per patient-year, with a biphasic pattern for onset of peritonitis which demonstrated an initial peak at 3-4 weeks and a second peak starting at 10 weeks after PD commencement and still rising at 12 weeks. The findings from this study were strengthened by its large sample size whereby the cohort represented over half the total number of PD patients in Colombia. Furthermore, the cohort represented a diverse range of education levels (none 29.3\%; elementary school 36\%; high school diploma $26 \%$; technical diploma $3 \%$; university degree $5 \%$; postgraduate degree $1 \%$ ) and socioeconomic positions [6].

Harel and colleagues similarly examined EOP within 3 months of catheter insertion (as opposed to PD commencement) in a retrospective case-control study of patients commencing PD at a single Canadian centre between 1998 and 2004 [10]. A total of 21 cases of EOP were identified and matched with a control group of 40 patients according to age, gender and status of diabetes mellitus, although specific details of the matching process were not described. Despite the fact that the patients with EOP and their controls were comparable with respect to a limited number of demographic and clinical characteristics, EOP patients were significantly younger than control patients (58.8 \pm 15.3 versus $67.4 \pm 15.5$ years, respectively, $p=0.04$ ), suggesting that the matching process adopted by the investigators was suboptimal. EOP patients exhibited significantly higher rates of peritonitis (1.18 vs. 0.28 episodes per patient-year) and technique failure ( 0.28 vs. 0.18 per patient-year). Patients with a history of EOP were almost twice as likely to die or to transfer to haemodialysis (adjusted hazard ratio [HR] 1.48, 95\% confidence interval $[\mathrm{Cl}]$ 0.65-3.38) in a multivariable model adjusted for age and coronary artery disease. Findings from this study were limited by its small sample size, retrospective design, suboptimal matching process and limited covariate adjustment with a moderate risk of confounding by indication. Nonetheless, it is interesting to note that despite clinical and study design heterogeneity amongst these studies, the proportions of patients who developed peritonitis (approximately $5-7 \%$ of patients) and the observed peritonitis rates within 3 months of PD (either starting PD or from time of catheter insertion) were generally comparable.

There have been two studies where EOP was defined as peritonitis occurring within 6 months of PD commencement $[8,9]$. These were both retrospective, single-centre, observational studies conducted in China with some differences in patient populations. Wang et al. grouped 155 incident PD patients who were older than 65 years of age into early, late and no-peritonitis groups in their study [7]. Thirty-two patients (20\%) developed peritonitis within six months of treatment initiation, with rates reported as 0.51 episodes per patient-year in the EOP group, compared to 0.33 episodes per patient-year in the late group. In contrast, Feng et al. included all incident PD patients regardless of their age ( $n=189$ patients) [5]. Patients were then divided into two groups based on occurrence of EOP. The authors identified that $30 \%$ of their cohort developed peritonitis within the first 6 months of PD. Although they were more inclusive by including patients of all age groups, Feng and colleagues excluded patients who stopped PD prior to 3 months, which could have introduced the risk of selection bias. Both studies were limited by small sample sizes, and single-centre and retrospective observational study designs. However, the proportions of patients observed to experience EOP was reasonably consistent (20-30\%).

In contrast, three studies of EOP defined as peritonitis occurring within 12 months of PD commencement observed variable peritonitis rates [12-14]. For instance, Fourtounas et al. retrospectively analysed outcomes for 168 PD patients from a single site in Greece over 12 years between 1993 and 2004 and dichotomised cohorts within the study to early and late groups [13]. The "early" group consisted of patients developing peritonitis within the first 12 months of PD therapy commencement, while the "late" group consisted of patients developing peritonitis two years or more after commencement of PD. Twenty (12\%) patients developed peritonitis in the first 12 months, whilst 27 (16\%) patients developed peritonitis in the "late" group with longer mean duration to first episode of peritonitis ( $46.3 \pm 17.6$ months compared to $5.7 \pm 3.2$ months). Over the 12-year follow up period, the reported peritonitis rate in the "early" group was 0.81 episodes per patient-year, which was significantly higher, compared to the "late" group (0.26 episodes per patient-year). Pulliam et al. reported on first year outcomes of incident PD patients across 466 dialysis units in the United States ( $n=1677$ ) [14]. This has been one of the largest studies to date, with $27.6 \%$ of their patients developing at least one episode of peritonitis within 12 months. The highest risk of peritonitis was in the first 6 months of PD (20\% of patients), compared to the latter 6 months of the study, which suggests that 6 months may be a more appropriate definition of EOP. This is consistent with a recently conducted analysis of the Australia and New Zealand Dialysis and Transplant (ANZDATA) Registry involving over 9000 patients across multiple sites in Australia, 
which observed a similar proportion of patients with peritonitis within the first 12 months of PD (27\%) [12].

In summary, despite variability in clinical settings, participant characteristics and definitions of EOP, the incidence of EOP has been generally comparable at both the 3-and 6-month time periods, $\sim 5-7 \%$ of PD patients affected by EOP by 3 months and $20-29 \%$ of patients affected by EOP by 6 months. However, findings have been more variable amongst the studies using a 12-month definition of EOP where $12-27 \%$ of patients are affected by EOP [5-7,9-11,13,14]. Some of these differences may be attributable to regional variations in background peritonitis rates (e.g. 0.26 episodes per patient year compared to 0.42 episodes per patient year).

\section{Importance of EOP}

Despite variably adopted definitions of what constitutes EOP, it is increasingly recognised as an important risk factor for poor patient outcomes. Feng and colleagues conducted a retrospective observational study from a single centre in China [5]. This study included all incident PD patients who commenced PD between 2004 and 2013 in their unit and divided patients into two groups based on occurrence of EOP, which was defined as peritonitis within the first 6 months of starting PD (EOP $n=55$; non-EOP $n=134)$. Even though all patients in this study developed peritonitis at some stage during their PD, those who developed peritonitis within the first 6 months of PD were at an increased risk of technique failure and mortality [5]. In fact, the occurrence of EOP in this group independently predicted technique failure ( $\mathrm{HR}$ 2.03, 95\% $\mathrm{Cl}$ 1.09-3.80, $\mathrm{p}=0.03$ ) and mortality (HR 1.69, 95\% Cl 1.12-2.87, $\mathrm{p}=0.002$ ) in a multivariable model adjusted for age, serum albumin, diabetes mellitus, Creactive protein and residual renal function [5]. Various studies have also noted subsequent higher peritonitis rates amongst EOP patients by two- to four-fold compared to non-EOP patients $[4,5,10]$. Although prevention of any peritonitis episode is likely to confer the most optimal patient outcome, this is not realistically feasible. However as Hsieh and colleagues have shown, a delay in onset of EOP may lead to clinically meaningful improvements in outcomes. In their study, they were able to demonstrate that every 1-month increase in the time to first peritonitis from start of PD can lead to a $2 \%$ decreased risk of technique failure and $3 \%$ reduction in the risk of patient mortality [11]. These results suggest that EOP is an important risk factor for adverse patient outcomes, and prevention or delay of its onset may lead to important improvement in patient-level outcomes.

\section{Risk Factors for EOP}

General risk factors for peritonitis have been extensively studied. They can be broadly grouped into modifiable and nonmodifiable types (Tables $\mathbf{1}$ and $\mathbf{2}$ ). It is however uncertain whether these general PD peritonitis risk factors are similarly applicable to EOP. Some of the studies on EOP have described risk factors, although they have been inconsistently examined with variable findings.

Table 1: Modifiable risk factors for general peritonitis and early onset peritonitis.

\begin{tabular}{|c|c|c|}
\hline Modifiable risk factors & General Peritonitis & Early onset Peritonitis \\
\hline Obesity & Yes [32-34] & Yes $[34]^{a}$ \\
\hline Smoking & Yes [33] & Not examined \\
\hline Living distantly from PD unit & Yes $[17,35]$ & Yes $[12]^{b}$ \\
\hline Depression & Yes $[36,37]$ & Not examined \\
\hline Hypoalbuminaemia & Yes [38,39] & Yes $[5-7,9]^{\mathrm{c}}$ \\
\hline Systemic antimicrobials at time of peritoneal dialysis catheter insertion & Yes [24] & Yes [24] $]^{\mathrm{d}}$ \\
\hline Diabetes & Yes $[33,34]$ & Yes $[11,12,14]^{\mathrm{e}}$ \\
\hline Nasal staphylococcus aureus carrier status & Yes [40] & Not examined \\
\hline Absence of vitamin D supplementation & Yes [39] & Not examined \\
\hline Prior haemodialysis & Yes [41] & Not examined \\
\hline PD against patient's choice & Yes $[42,43]$ & Not examined \\
\hline Biocompatible fluids & Yes [44] & Not examined \\
\hline Patient training & Yes $[20,45,46]$ & Not examined \\
\hline Medical procedures e.g. colonoscopy & Yes [47] & Not examined \\
\hline \multicolumn{3}{|c|}{$\begin{array}{l}\text { aNot consistently supported [5,6,11]; b Not consistently supported [6]; ' Not consistently supported [11,14]; }{ }^{\mathrm{d}} \text { Not consistently supported [24]; e Not consistently supported } \\
{[5-7,9,10]}\end{array}$} \\
\hline
\end{tabular}


Age

Age of PD patients has been one of the most frequently examined risk factors for EOP. Age is postulated to pose a risk to PD patients by mechanisms of reduced visual acuity, reduced manual dexterity leading to errors with connection, or potentially because older patients may have cognitive impairment or are more likely to be frail or co-morbid. Wang et al. reported a significant difference in the age of PD patients in their study whereby EOP patients were significantly older than those with late peritonitis episodes $(75.6 \pm 7.32$ vs. $72.6 \pm 5.8$ years, $p<0.05$ ) [7]. However, this was based on differences in patient characteristics at baseline of their observational study, which places the outcome at risk of bias from other confounding factors. For instance, similar methodology was adopted by other studies with contradictory findings whereby the mean ages of EOP patients were significantly younger than non-EOP patients $[5,6]$. Subsequent studies by Vargas (HR 1.00, 95\% Cl 0.99-1.01, $\mathrm{p}=0.58$ [6]), Wu (HR 1.01, 95\% Cl 1.00-1.03, $p=0.1$ [9]) and Pulliam (Relative risk [RR], per 10-y older, 0.98, 95\% Cl 0.93-1.04, $p=0.6$ [14]) were not able to observe any significant association between age and risk of EOP in their multivariable models. Therefore, although there is biological plausibility that increasing age may place patients at an increased risk of EOP, studies have not been able to confirm such an association.

Table 2: Non-modifiable risk factors for general peritonitis and early onset peritonitis.

\begin{tabular}{|l|l|l|}
\hline Non-modifiable risk factors & General Peritonitis & EOP \\
\hline Older age & Yes [33,41] & Yes [7,11,12] $^{\text {a }}$ \\
\hline Female sex & Yes [15,48,49] & No \\
\hline Previous exit-site infection & Yes [50,51] & Yes [9] \\
\hline $\begin{array}{l}\text { Indigenous or black racial } \\
\text { origin }\end{array}$ & $\begin{array}{l}\text { Yes } \\
{[16,33,34,39,52]}\end{array}$ & Yes [12,14] \\
\hline Poor residual kidney function & Yes [53] & Not examined \\
\hline Lower socioeconomic status & Yes [19,54] & No [6,9] \\
\hline Coronary artery disease & Yes [16,33] & Not examined \\
\hline Chronic lung disease & Yes [33] & Not examined \\
\hline Hypertension & Yes [16] & No [6,11] \\
\hline Pets & Yes [55] & Not examined \\
\hline
\end{tabular}

aNot consistently supported [5-7,9,10,14]; b Not consistently supported $[6,10]$

\section{Race}

Black and indigenous races have been found to be significantly associated with increased risks of EOP in some studies [7,13], which is consistent with the general peritonitis literature [14-18]. However, an association between racial origin and an increased risk of EOP has not been shown by other studies $[6,10]$. Moreover, the majority of studies to date have not examined race as a risk factor of EOP $[6,8-10,19]$, which may indeed be less relevant in countries such as China where almost all patients are of Asian race. Black or indigenous races have been variably reported to be associated with an increased risk of general peritonitis $[15,16]$, partially ascribed to established poorer health outcomes across Indigenous dialysis patients, compounded by remoteness and poor health and housing infrastructure [17].

\section{Socioeconomic position and remoteness}

Socioeconomic position and geographic remoteness have been variably associated with risk of PD peritonitis [16-20], partially attributed to impaired access to health care providers. Associations between socioeconomic positions and EOP have only been examined in a few studies $[10,11]$, with no evidence to suggest a significant association. Importantly, a study conducted in Colombia in which over half of their cohort were categorised into the two lowest tiers of socioeconomic stratification, as defined by the Columbian bureau of statistics (DANE), observed one of the lowest EOP rates ( 0.23 episodes per patient-year) [6]. Whilst educational level and literacy can be seen to be related to socioeconomic position, education level has been shown to negatively influence peritonitis risk even after adjustment for socio-economic position and comorbidities, a finding which will be relevant when discussing future avenues for EOP risk factor modification [19].

\section{Diabetes}

Patients with diabetes mellitus may be at an increased risk of developing EOP due to their predisposition toward infection in general rather than a specific risk factor for peritonitis $[6,21,22]$. Other proposed mechanisms for diabetes-related risk include reduced manual dexterity or visual impairment due to microvascular complications of diabetes $[23,24]$. Several studies have demonstrated diabetes to be a risk factor for EOP $[6,7]$, including one of the largest multi-centre studies to date (RR $1.18,95 \% \mathrm{Cl} 1.00-1.40, p=0.05$ [14]). However, many other studies have failed to demonstrate any association between diabetes status and the risk of EOP $[5-7,9,10]$.

\section{Obesity}

Obesity has been shown to be associated with an increased risk of peritonitis, possibly through physical factors such as exit site contamination due to location under abdominal pannus, or lack of visibility of exit-site for ongoing maintenance [21]. Increase in body mass index (BMI) has been identified as a risk factor for EOP by several groups $[9,12,14]$. However, others have reported low BMI as a risk factor for EOP [7]. Interestingly, in Wang et al. study, the mean BMI of patients categorised into non-peritonitis, early peritonitis and late peritonitis groups were relatively low, measured at $22.4 \pm 2.8,20.5 \pm 3.0$ and $21.8 \pm 2.8$ $\mathrm{kg} / \mathrm{m}^{2}$, respectively. Therefore, 'low' BMI as a risk factor for EOP in this study may in fact be a reflection of poor physical status, rather than a true 'risk' imposed by lean body mass. However, as the investigators only provided comparisons using baseline characteristic data, it is uncertain whether this association would still be present if a model was fitted including variables such as co-morbidities and age. 


\section{Hypoalbuminaemia}

These hypotheses are further supported by hypoalbuminaemia, which has been recognised as an important risk factor for both EOP and general peritonitis [6,9-11]. Serum albumin is often referenced as a marker of malnutrition status or inflammatory response, and low levels may represent 'poorer' health status indicating an increased risk of adverse health outcomes, such as EOP [5-7,9].

\section{Peripheral vascular disease}

Peripheral vascular disease was reported by Pulliam as a risk factor for EOP only when modeling for repeated episodes of peritonitis (RR 1.36, 95\% Cl 1.01-1.83, p=0.04) [14]. This has not been examined in the general peritonitis literature previously.

\section{Others}

Exit site infection has not been studied in detail as a risk factor of EOP. However, it is biologically plausible that exit site infection can increase risk of peritonitis. Wu and colleagues were able to demonstrate in their cohort that a prior history of exit site infection significantly increased the risk of subsequent EOP (odds ratio [OR] 4.14, 95\% Cl 2.45-7.00, p<0.001) in a multivariable model adjusted for age, gender, BMI, education status, diabetes mellitus and hypoalbuminaemia [9] .

\section{Preventative Strategies}

General preventive strategies are laid out in the International Society for Peritoneal Dialysis (ISPD) guidelines on reducing peritonitis [1]. These include topical antimicrobial use at catheter exit site and fungal prophylaxis during antibiotic therapy. The role of prophylactic systemic antimicrobials given at the time of peritoneal dialysis catheter insertion in preventing EOP is less clear. The ISPD guidelines refer to two small trials and a systematic review which demonstrate a reduction in early peritonitis with systemic antimicrobials at the time of peritoneal dialysis catheter insertion [22-24], however these trials define early peritonitis as within (at the most) 28 days of catheter insertion. Patients may not actually have commenced on PD treatment within this time period, which differs from published EOP studies to date in which the majority have specifically excluded patients within 3 months of catheter insertion $[5,7,9]$ or only considered peritonitis episodes after PD commencement $[6,11,13]$. As the exposure duration and timing of peritonitis defined by these studies differ, the role of prophylactic systemic antibiotic administration on preventing EOP remains uncertain. Furthermore a recently published Cochrane Systematic Review of this topic [25] references only a single study out of 39 which provides low quality evidence for intravenous vancomycin in reducing the risk of early peritonitis. Thus, it can be seen that EOP-specific preventative factors have been poorly elucidated. However, as $\mathrm{Wu}$ and colleagues have clearly demonstrated, a delay in onset of EOP has clinically important benefits at the patient level [9]. Therefore, strategies that can be implemented to prevent or delay the onset of EOP should be considered. One of these strategies is consideration of re-training. Studies have demonstrated that PD technique progressively worsens over time, often in spite of patients' best intentions [20]. Handwashing, technique recall, protocol adherence, and exit-site care have all been shown to progressively deteriorate and thus increase the risk of peritonitis [20,26-28]. The importance of retraining was well described by Russo and colleagues [20] who utilised a questionnaire to assess PD patients' knowledge about PD infection, medication use and PD procedures. They demonstrated that, after a mean of 33 months on PD, $47 \%$ of patients answered incorrectly. The authors then proposed a framework identifying 'at-risk' individuals based on knowledge testing and found $30 \%$ of patients qualified as needing retraining, both in the domains of infection control and medication use. Of note, re-training seemed to be indicated in a biphasic fashion, with a group at the beginning of their treatment (within 18 months of commencement) and then a group considered 'established' on dialysis (36 months of treatment or more). This biphasic pattern is especially relevant to EOP. Re-training could thus be considered prior to the 'peak' risk period of EOP, which appears to be reached by 6 months after PD commencement [5-7,9-11,13,14]. The ISPD guidelines further suggest retraining post 'triggering' events, such as exit site infection, catheter infection or episode of peritonitis; they additionally suggest re-training following changes in equipment, interruption in PD, hospitalisation, or changes in underlying patient physiology, such as visual changes or change in dexterity [1]. Re-training may therefore represent a key area for future preventative studies.

In addition, PD care providers should consider adopting specifically tailored preventative strategies for patients with recognised risk factors for EOP, such as optimising nutrition and glycaemic control, ensuring appropriate placement of PD catheter exit site in obese patients [21], and undertaking home visits earlier in training in the case of geographical isolation [16]. Although there has not been a study to demonstrate reduction in peritonitis risk from optimisation of diabetes management, optimal glycaemic control has been shown to reduce the risk of peri-operative infection [29].

Finally, when faced with risks associated with elderly or comorbid patients, assisted PD has been proposed as a further avenue to prevent PD-related complications [30,31]. However, its impact on reducing peritonitis risks has not been demonstrated. For example, Benabed et al. examined the risk of peritonitis in diabetic patients within a French nurse-assisted PD program, in which catheter connection and disconnection was performed by private nurses at the patient's home [30]. They found no difference in incidence of peritonitis between groups, a finding consistent with other studies on assisted PD from Taiwan [31].

\section{Future Directions}

Early onset peritonitis has been demonstrated through multiple reviews to represent a risk factor for adverse outcomes for PD patients. However, the definitions used by studies to date have been variable such that this heterogeneity renders the drawing of systematic conclusions difficult. More research is needed to identify 'at risk' time periods, specific risk factors and effective preventative strategies. 


\section{Disclosures}

All the authors have declared no competing interest.

\section{References}

1. Piraino B, Bernardini J, Brown E, Figueiredo A, Johnson DW, et al. (2011) ISPD position statement on reducing the risks of peritoneal dialysis-related infections. Perit Dial Int. 31: 614-630.

2. Zhang L, Hawley CM, Johnson DW (2016) Focus on peritoneal dialysis training: working to decrease peritonitis rates. Nephrol Dial Transplant 31: 214-222.

3. Brown MC, Simpson K, Kerssens JJ, Mactier RA (2009) Encapsulating peritoneal sclerosis in the new millennium: a national cohort study. Clin J Am Soc Nephrol 4: 1222-1229.

4. Cho Y, Johnson DW (2014) Peritoneal dialysis-related peritonitis: towards improving evidence, practices, and outcomes. Am J Kidney Dis 64: 278-289.

5. Feng S, Wang Y, Qiu B, Wang Z, Jiang L, et al. (2016) Impact of early-onset peritonitis on mortality and technique survival in peritoneal dialysis patients. Springerplus 5: 1676.

6. Vargas E, Blake PG, Sanabria M, Bunch A, Lopez P, et al. (2017) Early Peritonitis in a Large Peritoneal Dialysis Provider System in Colombia. Perit Dial Int 37: 30-34.

7. Wang Z, Jiang L, Feng S, Yang L, Jiang S, et al. (2015) Early peritonitis is an independent risk factor for mortality in elderly peritoneal dialysis patients. Kidney Blood Press Res 40: 298-305.

8. Gan HC, Lee EJ, Tay MM, Yusuf N (2003) A study on early onset peritonitis in CAPD patients. Singapore Med J 44: 138-40.

9. Wu H, Huang R, Yi C, Wu J, Guo Q, et al. (2016) Risk Factors for Early-Onset Peritonitis in Southern Chinese Peritoneal Dialysis Patients. Perit Dial Int. 36: 640-646.

10. Harel Z, Wald R, Bell C, Bargman JM (2006) Outcome of patients who develop early-onset peritonitis. Adv Perit Dial 22: 46-49.

11. Hsieh YP, Wang SC, Chang CC, Wen YK, Chiu PF, et al. (2014) The negative impact of early peritonitis on continuous ambulatory peritoneal dialysis patients. Perit Dial Int 34: 627-635.

12. See EJ, Johnson DW, Hawley CM, Pascoe EM, Darssan D, et al. (2017) Early Peritonitis and its outcome in incident peritoneal dialysis patients. Perit Dial Int (In press).

13. Fourtounas $C$, Savidaki E, Dousdabanis $P$, Hardalias A, Kalliakmani $P$, et al. (2006) Peritonitis during the first year after commencement of peritoneal dialysis has an impact on technique survival and patient morbidity. Adv Perit Dial 22: 50-54.

14. Pulliam J, Li NC, Maddux F, Hakim R, Finkelstein FO, et al. (2014) First-year outcomes of incident peritoneal dialysis patients in the United States. Am J Kidney Dis. 64: 761-769.

15. Nessim SJ, Bargman JM, Austin PC, Nisenbaum R, Jassal SV (2009) Predictors of peritonitis in patients on peritoneal dialysis: results of a large, prospective Canadian database. Clin J Am Soc Nephrol 4: $1195-1200$.

16. Lim WH, Boudville N, McDonald SP, Gorham G, Johnson DW, et al. (2011) Remote indigenous peritoneal dialysis patients have higher risk of peritonitis, technique failure, all-cause and peritonitisrelated mortality. Nephrol Dial Transplant 26: 3366-3372.

17. Cho Y, Badve SV, Hawley CM, McDonald SP, Brown FG, et al. (2012) The effects of living distantly from peritoneal dialysis units on peritonitis risk, microbiology, treatment and outcomes: a multicentre registry study. BMC Nephrol 13: 41.

18. Tang W, Grace B, McDonald SP, Hawley CM, Badve SV, et al. (2015) Socio-Economic Status and Peritonitis in Australian NonIndigenous Peritoneal Dialysis Patients. Perit Dial Int 35: 450-459.

19. Martin LC, Caramori JC, Fernandes N, Divino-Filho JC, Pecoits-Filho R, et al. (2011) Geographic and educational factors and risk of the first peritonitis episode in Brazilian Peritoneal Dialysis study (BRAZPD) patients. Clin J Am Soc Nephrol 6: 1944-1951.

20. Russo R, Manili L, Tiraboschi G, Amar K, De Luca M, et al. (2006) Patient re-training in peritoneal dialysis: why and when it is needed. Kidney Int Suppl 2006: S127-S132.

21. Crabtree JH (2006) Selected best demonstrated practices in peritoneal dialysis access. Kidney Int Suppl 2006: S27-S37.

22. Gadallah MF, Ramdeen G, Mignone J, Patel D, Mitchell L, et al. (2000) Role of preoperative antibiotic prophylaxis in preventing postoperative peritonitis in newly placed peritoneal dialysis catheters. Am J Kidney Dis 36: 1014-1019.

23. Bennett-Jones DN, Martin J, Barratt AJ, Duffy TJ, Naish PF, et al. (1988) Prophylactic gentamicin in the prevention of early exit-site infections and peritonitis in CAPD. Adv Perit Dial 4: 147-150.

24. Strippoli GF, Tong A, Johnson D, Schena FP, Craig JC (2004) Antimicrobial agents to prevent peritonitis in peritoneal dialysis: a systematic review of randomized controlled trials. Am J Kidney Dis 44: 591-603.

25. Campbell DMD, Craig JC, Johnson DW, Tong A, Strippoli GFM (2017) Antimicrobial agents for preventing peritonitis in peritoneal dialysis patients. Cochrane Database Syst Rev 4: CD004679.

26. Troidle L, Finkelstein F (2006) Treatment and outcome of CPDassociated peritonitis. Ann Clin Microbiol Antimicrob 5: 6 .

27. Piraino B, Bernardini J, Brown E, Figueiredo A, Johnson DW, et al. (2011) ISPD-position statement on reducing the risks of peritoneal dialysis-related infections. Perit Dial Int 31: 614-630.

28. Cho Y, Badve SV, Hawley CM, McDonald SP, Brown FG, et al. (2013) Effects of Climatic Region on Peritonitis Risk, Microbiology, Treatment, and Outcomes: a Multicenter Registry Study. Perit Dial Int 33: 75-85.

29. Hanazaki K, Maeda H, Okabayashi T (2009) Relationship between perioperative glycemic control and postoperative infections. World J Gastroenterol 15: 4122-4125.

30. Benabed A, Bechade C, Ficheux M, Verger C, Lobbedez T (2016) Effect of assistance on peritonitis risk in diabetic patients treated by peritoneal dialysis: report from the French Language Peritoneal Dialysis Registry. Nephrol Dial Transplant 31: 656-662.

31. Cheng CH, Shu KH, Chuang YW, Huang ST, Chou MC, et al. (2013) Clinical outcome of elderly peritoneal dialysis patients with assisted care in a single medical centre: a 25 year experience. Nephrology (Carlton) 18: 468-473.

32. Lim WH, Johnson DW, McDonald SP (2005) Higher rate and earlier peritonitis in Aboriginal patients compared to non-Aboriginal patients with end-stage renal failure maintained on peritoneal dialysis in Australia: analysis of ANZDATA. Nephrology (Carlton) 10: 192-197.

33. McDonald SP, Collins JF, Rumpsfeld M, Johnson DW (2004) Obesity is a risk factor for peritonitis in the Australian and New Zealand peritoneal dialysis patient populations. Perit Dial Int 24: 340-346. 
34. Ghali JR, Bannister KM, Brown FG, Rosman JB, Wiggins KJ, et al. (2011) Microbiology and outcomes of peritonitis in Australian peritoneal dialysis patients. Perit Dial Int 31: 651-662.

35. Lim WH, Boudville N, McDonald SP, Gorham G, Johnson DW, et al. (2011) Remote indigenous peritoneal dialysis patients have higher risk of peritonitis, technique failure, all-cause and peritonitisrelated mortality. Nephrol Dial Transplant 26: 3366-3372.

36. Juergensen PH, Wuerth DB, Juergensen DM, Finkelstein SH, Steele $T E$, et al. (1997) Psychosocial factors and clinical outcome on CAPD. Adv Perit Dial 13: 121-124.

37. Troidle L, Watnick S, Wuerth DB, Gorban-Brennan N, Kliger AS, et al. (2003) Depression and its association with peritonitis in longterm peritoneal dialysis patients. Am J Kidney Dis 42: 350-354.

38. Chow KM, Szeto CC, Leung CB, Kwan BC, Law MC, et al. (2005) A risk analysis of continuous ambulatory peritoneal dialysis-related peritonitis. Perit Dial Int 25: 374-379.

39. Kerschbaum J, Konig P, Rudnicki M (2012) Risk factors associated with peritoneal-dialysis-related peritonitis. Int J Nephrol 2012: 483250.

40. Wanten GJ, van Oost P, Schneeberger PM, Koolen MI (1996) Nasal carriage and peritonitis by Staphylococcus aureus in patients on continuous ambulatory peritoneal dialysis: a prospective study. Perit Dial Int 16: 352-356.

41. Nessim SJ, Bargman JM, Austin PC, Story K, Jassal SV (2009) Impact of age on peritonitis risk in peritoneal dialysis patients: an era effect. Clin J Am Soc Nephrol 4: 135-141.

42. Oygar DD, Yalin AS, Altiparmak MR, Ataman R, Serdengecti $K$ (2011) Obligatory referral among other factors associated with peritonitis in peritoneal dialysis patients. Nefrologia 31: 435-440.

43. Rodriguez-Carmona A, Perez Fontan M, Garcia Falcon T, Fernandez Rivera C, Valdes F (1999) A comparative analysis on the incidence of peritonitis and exit-site infection in CAPD and automated peritoneal dialysis. Perit Dial Int 19: 253-258.

44. Johnson DW, Brown FG, Clarke M, Boudville N, Elias TJ, et al. (2012) The effects of biocompatible compared with standard peritoneal dialysis solutions on peritonitis microbiology, treatment, and outcomes: the balANZ trial. Perit Dial Int 32: 497-506.
45. Chow KM, Szeto CC, Law MC, Fun Fung JS, Kam-Tao Li P (2007) Influence of peritoneal dialysis training nurses' experience on peritonitis rates. Clin J Am Soc Nephrol 2: 647-652.

46. Holloway M, Mujais S, Kandert M, Warady BA (2001) Pediatric peritoneal dialysis training: characteristics and impact on peritonitis rates. Perit Dial Int 21: 401-404.

47. Yip T, Tse KC, Lam MF, Cheng SW, Lui SL, et al. (2007) Risks and outcomes of peritonitis after flexible colonoscopy in CAPD patients. Perit Dial Int 27: 560-564.

48. Ros S, Remon C, Qureshi AR, Quiros P, Lindholm B, et al. (2013) Increased risk of fatal infections in women starting peritoneal dialysis. Perit Dial Int 33: 487-494.

49. Perez Fontan M, Rodriguez-Carmona A, Garcia-Naveiro R, Rosales $M$, Villaverde $P$, et al. (2005) Peritonitis-related mortality in patients undergoing chronic peritoneal dialysis. Perit Dial Int 25: 274-284.

50. Lloyd A, Tangri N, Shafer LA, Rigatto C, Perl J, et al. (2013) The risk of peritonitis after an exit site infection: a time-matched, casecontrol study. Nephrol Dial Transplant 28: 1915-1921.

51. van Diepen AT, Tomlinson GA, Jassal SV (2012) The association between exit site infection and subsequent peritonitis among peritoneal dialysis patients. Clin J Am Soc Nephrol 7: 1266-1271.

52. Lim WH, Johnson DW, McDonald SP (2005) Higher rate and earlier peritonitis in Aboriginal patients compared to non-Aboriginal patients with end-stage renal failure maintained on peritoneal dialysis in Australia: analysis of ANZDATA. Nephrology (Carlton) 10: 192-197.

53. Han SH, Lee SC, Kang EW, Park JK, Yoon HS, et al. (2012) Reduced residual renal function is associated with endothelial dysfunction in patients receiving peritoneal dialysis. Perit Dial Int 32: 149-158.

54. Farias MG, Soucie JM, McClellan W, Mitch WE (1994) Race and the risk of peritonitis: an analysis of factors associated with the initial episode. Kidney Int 46: 1392-1396.

55. Schiller B, Alcaraz M, Hadley K, Moran J (2011) Peritonitis and zoonosis: your best friend sometimes isn't! Perit Dial Int 31: 127-130. 